\title{
Overactive bladder: Symptom complex or separate entity?
}

\author{
Sender Herschorn, MD, FRCSC \\ Division of Urology, University of Toronto, and Martin Barkin Chair in Urological Research and attending urologist, Sunnybrook Health Sciences Centre, Toronto, ON
}

Cite as: Can Urol Assoc J 2011;5(5Supp|2):S152-S154; D01:10.5489/cuaj.11210

$\mathrm{n}$ addition to the series of lectures summarized in the articles on the preceding pages, the 2011 Canadian Urology

Forum also included a series of workshops intended to help answer the question: "Does overactive bladder (OAB) exist as a separate entity or is it a symptom complex that may be associated with other conditions?"

In an effort to arrive at an answer to this question, the participants were divided into groups and asked to examine a particular subset of patients with OAB. The subsets were: females, males, neurogenic and elderly. In each of these workshops, the participants were asked to answer the following questions:

- What is the most likely underlying cause?

- $\quad$ Are there unique phenotypes that can be identified clinically that are linked to a common underlying pathophysiology?

- What is the likelihood of associated disorders of the outlet or pelvic floor?

- What are the most effective treatments?

Each of the groups then reported their answers back to the entire group of participants, after which time they were asked to vote on the key question. This review provides a summary of the discussions that took place in each group and concludes with the results of the vote.

\section{$O A B$ in females}

\section{Underlying causes}

There was a great deal of debate about the most likely underlying causes of $\mathrm{OAB}$ in women. At the end of the workshop, it was concluded that this question was not possible to answer, as there is an extensive list of potential underlying causes.

\section{Phenotypes}

One could describe several different phenotypes of OAB, based on any identified underlying cause (e.g., gynecologic such as pelvic organ prolapse, medical, neurological). One could also identify some particular groups of idiopathic OAB that one might describe as phenotypes (e.g., very young women with severe urgency and urgency incontinence). However, given that we cannot reliably link these subgroups to a particular underlying cause, labelling them as phenotypes does not serve any useful purpose.

\section{Likelihood of associated disorders}

Among women, there are some associated disorders of bladder outlet or pelvic floor that may lead to symptoms of OAB. Prolapse, urethral instability and perhaps even irritable bowel were the potential associations discussed during this workshop.

\section{Most effective treatments}

Optimal treatment for women with $\mathrm{OAB}$ involves a combination of lifestyle and behavioural modification with drug therapy. The workshop group concluded that although there are not yet any data for botulinum toxin as a first-line therapy, this agent may be the most effective of the available options.

\section{$O A B$ in males}

\section{Underlying causes}

The workshop presenter for this group identified a number of potential underlying causes of OAB in males, broken down into myogenic, neurogenic, idiopathic, habits and social subgroups. These top-line groups were then further broken down into more particular causes. Among myogenic 
causes, for example, the discussion identified bladder ischemia, obstruction (e.g., that may be associated with bladder wall thickening) and aging as potential contributing factors. Among neurogenic causes, they listed central (e.g., stroke), peripheral (e.g., diabetes, surgery) and the aging brain. Among the habits and social causes, fluid and caffeine intake were cited, as was cigarette smoking.

\section{Phenotypes}

This group of participants identified specific clinical phenotypes based on the particular underlying mechanisms. For ischemia, older age and potentially severe vasculopathy were identified as potential clinical phenotypes. For obstruction, the clinical phenotypes were BPH, tight bladder neck, strictures and dysfunctional voiders.

Among the neurogenic causes, some of the associated specific phenotypes are patients with diabetes, stroke survivors, individuals with Parkinson's disease, and those who have undergone surgery. One phenotype associated with idiopathic $O A B$ is the type $A$ young man who cannot relax. Metabolic syndrome has also been related to $O A B$, and may be a separate phenotype.

\section{Likelihood of associated disorders}

The workshop participants identified a number of abnormalities that can be associated with $\mathrm{OAB}$, including tight bladder neck, BPH, stricture, dysfunctional voiding (i.e., someone who cannot relax his pelvic floor, with no neurologic cause), chronic prostatitis and severe constipation.

\section{Most effective treatments}

The participants clearly stated that for $O A B$ in men, there is no one therapeutic strategy that can be applied across the spectrum of patients. The most effective treatments for men with $\mathrm{OAB}$ were, therefore, once again addressed in the context of the different types of patients described above.

For myogenic causes, the participants recommended management with a combination of behavioural modification and anticholinergic medication. For those with obstruction, it was suggested that alpha-blockade with or without 5-alpha-reductase inhibitors would be the best option. For bladder ischemia, although there was no consensus on the best management, suggested options were PDE-5 inhibitors, 5-alpha-reductase inhibitors and/or anticholinergics in conjunction with lifestyle modification. Surgical procedures (e.g., transurethral resection of the prostate [TURP], transurethral bladder neck incision) may also play a role. For dysfunctional voiders, some benefit may be obtained through behaviour training and biofeedback training.
For OAB symptoms associated with specific medical conditions (e.g., Parkinson's disease, diabetes), optimal management of the underlying condition is recommended and this may help improve the $O A B$ symptoms.

\section{$O A B$ in the elderly}

\section{Underlying causes}

There are a number of potential underlying causes of $O A B$ in the elderly, as identified by the participants in this workshop. Ischemia, oxidative stress and endothelial dysfunction are associated with the aging process and can occur at all levels, including the bladder, pelvic floor, and the brain. Ischemia, in particular, may cause microinfarcts that can affect areas of critical inhibition. Neural apoptosis and degeneration may also play a role with advancing age, and this can also have an impact both centrally and locally.

Specific neurologic conditions (e.g., Parkinson's Disease) that are more common in elderly patients are also associated with increased risk of $\mathrm{OAB}$ symptoms. Chronic infections may also play a role. BPH may also be accompanied by nerve transmission changes and ischemia. Other possible contributors that are more common in older patients include use of prescription medications (including polypharmacy), constipation, decreased mobility, and dietary changes.

\section{Phenotypes}

The participants in this workshop identified some clinical phenotypes of $O A B$ in the elderly. Obesity seems to represent a particular phenotype. Certain demographic groups have also been identified as having an elevated risk and may be described as clinical phenotypes (e.g., African American women). The workshop also included a discussion regarding diet, concluding that those with a poor diet have an increased potential for $\mathrm{OAB}$ symptoms. Individuals who were late bedwetters in childhood may also be a clinical phenotype predisposed to nocturia in adulthood.

\section{Likelihood of associated disorders}

$\mathrm{OAB}$ in the elderly is indeed thought to be associated with outlet and/or pelvic floor disorders. The support for this contention comes from the observations from population-based studies and from reports of treatment. If one treats these potentially associated disorders (e.g., prolapse), the $O A B$ symptoms may improve. 
Herschorn

\section{Most effective treatments}

Conservative management, such as prompted voiding, can be effective for older patients, but it is resource intensive and not easily sustainable. For cognitively intact older patients, the workshop participants generally agreed that these individuals should be treated with the same approach used for younger adults. Among those who are cognitively impaired, however, one should be very cautious and start with low doses of anticholinergic agents. Some elderly patients may require higher doses of medication to achieve clinical benefit, but the dose should be titrated slowly. Botulinum toxin was regarded as a potential option in elderly patients, using low doses.

\section{Neurogenic OAB}

\section{Underlying causes}

The most likely underlying cause appears to be a neurogenic bladder, although there are also some suggestions that it is also centrally mediated. What the particular abnormality(ies) may be is not fully understood, but the participants focused their discussion on the increase in afferent firing as a result of the neurogenic changes to the bladder. They also discussed the possibility of secondary associated changes in the urothelium or the detrusor muscle itself.

There may also be differences in innervation or receptor distribution in neurogenic $O A B$. It appears that there are redistributions of various $\mathrm{M} 2$ and $\mathrm{M} 3$ receptors; TRPV1 and $\mathrm{P} 2 \mathrm{X}$ receptors might be upregulated in neurogenic bladders. There may also be changes related to suprapontine disruptions.

\section{Phenotypes}

Neurogenic $O A B$ does have clear clinical phenotypes related to the presence of individual neurologic conditions (e.g., Parkinson's Disease, spinal cord injury, suprapontine lesions).

\section{Likelihood of associated disorders}

The discussion about associated disorders included the recognition of certain primary diagnoses (e.g., detrusor sphincter dyssynergia) that are associated with $\mathrm{OAB}$ symptoms. With respect to $\mathrm{BPH}$, the participants also discussed the possibility that this condition may be affected by a change in innervation. The association of stress incontinence with neurogenic $\mathrm{OAB}$ changes was also discussed.

\section{Most effective treatments}

For neurogenic $O A B$, it appears based on the evidence compiled to date that botulinum toxin is likely to be the most effective treatment. However, at present, even though the data is not expansive, the majority of participants agreed that they would use first-line treatment with anticholinergic therapies in this patient population. There are also a number of intriguing potential candidates for drug therapy — including agents targeting central nervous pathways - which the participants felt provided some promise for future management.

\section{Is $O A B$ a separate entity? discussion and consensus}

The aim of the 2011 Canadian Urology Forum was to carefully examine the current state of knowledge in $O A B$ through a number of sessions led by some of the world's thought leaders on this subject. The reviews in this document have documented the presentations and discussions conducted during those sessions, including the elements of $O A B$ pathophysiology and the treatments that are used to manage this condition across a spectrum of patient populations.

And the conclusion of the Urology Forum, the participants were asked to reflect on all they had learned during the course of the educational sessions and vote on the central question of the conference: "Does overactive bladder $(\mathrm{OAB})$ exist as a separate entity or is it a symptom complex associated with other disease?"

Before the vote was conducted, the deliberations of the workshops were presented, discussed, and summarized. It was pointed out that there are multiple underlying causes and associations for $\mathrm{OAB}$; there are also different phenotypes and many different treatment modalities, some of which work better for some groups than for others.

These observations appear to have been taken into account by the majority of the participants, who overwhelmingly voted that $O A B$ is not a separate entity, but a symptom complex that may be associated with other conditions (final vote: 21 to 5). There was consensus that $\mathrm{OAB}$ needs to be redefined on this basis.

Competing interests: Dr. Herschorn has received consultant/speaker fees from Allergan, Astellas, American Medical Systems, Pfizer, Contura/Johnson and Johnson, Triton, Watson and Merck.

This paper has been peer-reviewed.

Correspondence: Dr. Sender Herschorn, Division of Urology, University of Toronto, Sunnybrook Health Sciences Centre, Rm. A309, 2075 Bayview Ave., Toronto ON M4N 3M5; s.herschorn@utoronto.co 